\title{
VALORACIÓN DE LA FUNCIÓN DEL TRACTO URINARIO INFERIOR EN EL PACIENTE PEDIÁTRICO PORTADOR DE REFLUJO VESICOURETERAL PRIMARIO
}

\author{
Rosa Martín-Crespo Izquierdo y Rafael Luque Mialdea.
}

Servicio de Cirugía Pediátrica. Urología Pediátrica. Unidad de Urodinámica. Complejo Hospitalario de Toledo. Toledo. España.

\begin{abstract}
Resumen.- OBJETIVOS: Los objetivos del trabajo son: Presentar una metodología de valoración perioperatoria de la función del tracto urinario inferior en el paciente con Reflujo Vésico Ureteral (RVU) Primario; Describir los patrones urodinámicos más frecuentes en los pacientes con RVU primario y su tratamiento en base al patrón urodinámico. Exponer los resultados de la valoración del tracto urinario inferior de un estudio prospectivo de una población pediátrica de 63 pacientes, portadores de RVU primario persistente, con el fin de ayudar a la mejor comprensión de la historia natural del RVU.
\end{abstract}

MÉTODOS: El estudio comprende una población pediátrica de 63 pacientes portadores de RVU primario grado IIV, 28 niñas y 35 varones, de edades comprendidas entre 6 meses y 15 años de edad, con una edad media global de 5,9 años. Dentro del protocolo de estudio sistemático RVU primario, se realiza un despistaje no invasivo de la presencia de malfunción del tracto urinario inferior (MTUI), del cual se seleccionan los pacientes que se beneficiarán de un estudio cistométrico completo. Variables estudiadas: edad, sexo, lateralidad y grado de RVU, y función renal, en relación con la función del tracto urinario inferior (TUI) al reclutamiento. Los resultados

Rosa Martín-Crespo

Servicio de Cirugía Pediátrica

Complejo Hospitalario de Toledo

Avda. Barber, 30

45004 Toledo. (España).

rmartinc@telefonica.net del estudio urodinámico se han valorado en relación a las variables: sintomatología urinaria y antecedente de infección del tracto urinario inferior (ITU).

RESULTADOS: Características diferenciales de la población estudiada: Edad media elevada Iglobal de 5,9 años; 7,7 años en las niñasl, porcentaje elevado de RVU de alto grado (59\%), de daño renal (52\%) y de MTUI (86\%), sin diferencias significativas entre varones y niñas. Asociación estadísticamente significativa entre: Grado de RVU y daño renal; presencia de MTUI y bilateralidad del RVU; sexo femenino y MTUI; y normalidad de la función del TUI y ausencia de daño renal. Predominio del patrón urodinámico tipo disfunción miccional en la niña y de la hiperactividad del detrusor en el varón. La sintomatología urinaria no difiere en los distintos patrones de alteración urodinámica. Solo la incidencia de ITU muestra diferencias significativas entre los distintos patrones urodinámicos, siendo mayor en las niñas con patrón urodinámico tipo disfunción miccional (micción no coordinada).

CONCLUSIONES: La realización de un despistaje no invasivo de la función del TUI mediante la historia miccional y fecal, el análisis urinario, la ecografía y la medida ecográfica del residuo postmiccional, permite la selección de los pacientes que se beneficiarán de un estudio urodinámico completo. La cistometría pediátrica aporta datos precisos sobre las fases de llenado y vaciado vesical, que facilitan el tratamiento específico de la MTUI. El estudio urodinámico cobra un especial significado en el pronóstico de la malformación urológica asociada en los niños portadores del RVU. Con esta sistemática de estudio de la función del TUI puede lograrse un diagnóstico y tratamiento precoz de los pacientes en riesgo y una adecuada selección de los pacientes que se beneficiarán de la farmacoterapia y/o uroterapia, con el fin de prevenir la progresión del daño renal y potenciar la curación definitiva del RVU.

Palabras clave: Reflujo vesico-ureteral. Función del tracto urinario inferior. Estudio urodinamico No invasivo. Cistometria pediátrica. Malfunción del tracto urinario inferior. Nefropatía por reflujo. 
Summary.- OBJECTIVES: To present a methodology of perioperative evaluation of lower urinary tract function in patients with primary VUR. To describe the most frequent urodynamic patterns in patients with primary VUR and their treatment. To show the results of a lower urinary tract evaluation in a prospective study in a pediatric population of 63 patients, with persistent primary VUR, with the aim to help to a better understanding of the natural history of VUR.

METHODS: The study includes a pediatric population of 63 patients with primary grade IIVV VUR, 28 girls and 35 boys, with ages between six months and 15 years (mean age 5.9 yr.). In the study protocol for the systematic study of primary vesicoureteral reflux we perform a non invasive screening for lower urinary tract dysfunction, which select patients that will benefit from a complete cystomanometry. Study variables: age, gender, VUR side and grade, and renal function, in relation with the lower urinary tract function at the time of recruitment. The results of urodynamics have been evaluated in relation to urinary symptoms and history of lower urinary tract infection.

RESULTS: Differential characteristics of the study population: high mean age loverall 5.9 yr.; 7.7 in girls), high percentage of high grade VUR (59\%), renal damage (52\%) and lower urinary tract dysfunction (86\%) without significant differences between boys and girls. Statistically significant associations between: VUR grade and renal damage; presence of lower urinary tract dysfunction and bilateral VUR; female gender and lower urinary tract dysfunction; and normal lower urinary tract function and absence of renal damage. The voiding dysfunction pattern was predominant in females and bladder hyperactivity in males. Urinary symptoms did not differ between the various patterns of urodynamic abnormalities. The incidence of UTI shows significant differences between the various urodynamic patterns, being more frequent in girls with type 4 urodynamic pattern (dysfunctional voiding).

CONCLUSIONS: The performance of a non invasive screening of lower urinary tract function by medical history, urine analysis, ultrasound and post void residual ultrasound evaluation enabled the selection of patients who would benefit from a complete urodynamic study. If there are symptoms or signs of lower urinary tract dysfunction it is recommended to perform a complete urodynamic study. Pediatric cystomanometry provides precise data about bladder filling and voiding phases, facilitating the specific treatment of the lower urinary tract dysfunction. The urodynamic study has a special significance in the prognosis of the urological malformation associated in children with VUR. With this systematic study of the lower urinary tract function early diagnosis and treatment of patients in risk may be achieved, as well as adequate selection of patients that will benefit from pharmacotherapy and/or urotherapy, with the aim to prevent renal damage progression and to potentiate definitive cure of VUR.

Keywords: Vesicoureteral reflux. Lower urinary tract function. Non invasive urodynamic study. Pediatric cystomanometry. Lower urinary tract dysfunction. Reflux nephropathy.

\section{INTRODUCCIÓN}

El conocimiento de la relación entre la obstrucción al flujo urinario y la alteración de la función del tracto urinario inferior ha tenido su mayor exponencial en los casos de obstrucción anatómica (válvulas de uretra) y funcional (vejiga neurógena), en las que el reflujo vesico-ureteral se considera secundario al malfuncionamiento vésico-uretral como consecuencia de la obstrucción. En la actualidad hay cada vez mas evidencias de que el reflujo vesicoureteral $(\mathrm{RVU})$ se asocia con frecuencia a una malfunción del tracto urinario inferior (MTUI) tanto en lactantes como en niños mayores, en ausencia de causa neurológica o estructural identificable; malfunción que parece tener un papel importante en la patogenia, génesis y/o perpetuación del RVU.

La MTUI representa una alteración funcional de la vejiga y la uretra, en sus dos fases de llenado vesical y vaciado vesical o miccional, con un amplio espectro de gravedad, y cuya significación clínica puede variar desde la enuresis a la insuficiencia renal.

El RVU en el lactante y niño mayor es considerado un factor de riesgo de desarrollo de infección del tracto urinario (ITU), nefropatía por RVU (daño renal congénito, progresivo o formación de cicatrices renales) e insuficiencia renal. La presencia de MTUI se ha relacionado, a su vez, con un mayor riesgo e incidencia de ITU, involucrada en la formación de nuevas cicatrices renales, disfunción anorrectal o fecal, y la persistencia de RVU, tanto en relación al retraso en su resolución espontánea como al fracaso en su curación o a la recidiva después del tratamiento quirúrgico (convencional o endoscópico) (Capozza, 2002 (1); Herndon, 2001 (2). Parece que el "Fenómeno RVU" no es una entidad patogénica homogénea sino un espectro heterogéneo de condiciones patológicas donde diferentes factores pueden operar en la génesis o perpetuación del RVU, ITU y nefropatía por RVU.

Independientemente de la causa subyacente, la resolución del RVU puede llevar meses o años; Una gran proporción de lactantes no sufrirán resolución del RVU con tratamiento conservador. Todavía hoy sigue la controversia en relación con los factores predictivos del pronóstico clínico del RVU. Existen varios factores pronósticos que se han involucrado en la predicción de la resolución espontánea del RVU (Yeung, 2006 (3), Sillen, 2007 (4). Entre estos factores, la MTUI presente al diagnóstico, se ha relacionado con una menor tasa de resolución espontánea del RVU. Así, históricamente, los pacientes portadores de RVU concomitante con MTUI, con elevada incidencia de ITU y daño renal, y reducida tasa de resolución espontánea, constituyen la mayoría de los pacientes pediátricos que han sido sometidos a tratamiento quirúrgico del RVU.

Los objetivos de este trabajo son:

- Presentar una metodología de estudio perioperatorio de la función del Tracto Urinario Inferior (TUI) en el paciente con RVU primario.

- Exponer los patrones urodinámicos más frecuentes en los pacientes con RVU primario y su tratamiento en base al patrón urodinámico.

- Exponer los resultados de un estudio prospectivo de una 
población pediátrica de 63 pacientes, portadores de RVU primario persistente, carente de atención urológica pediátrica previa, que relacionan la edad, sexo, lateralidad y grado de RVU, y función renal con la función del tracto urinario inferior, con el fin de ayudar a la mejor comprensión de la historia natural del RVU.

\section{VALORACIÓN PERIOPERATORIA DE LA FUNCIÓN DEL TRACTO URINARIO INFERIOR (TUI)}

Al inicio del estudio del RVU, y dentro del protocolo de estudio sistemático del RVU (Tabla I), se realiza un despistaje no invasivo de la presencia de MTUI, del cual se seleccionan los pacientes que se beneficiarán de un estudio cistométrico completo. La terminología empleada sigue los estándares recomendados por las Sociedades de Continencia Internacional (ICS) y de Continencia Pediátrica Internacional (ICCS).

\section{ESTUDIO NO INVASIVO DE LA FUNCIÓN DEL TUI:}

\section{Historia clínica}

\section{- Historia miccional}

Historia de adquisición de la continencia; entrenamiento y edad de adquisición de continencia urinaria y fecal, diurna y nocturna. Comportamiento y hábito miccional, útil para detectar población en riesgo de MTUI.

\section{- Síntomas de MTUI:}

A. Síntomas durante la fase de llenado

I. Frecuencia miccional diurna (aumentada o disminuida)

II. Incontinencia urinaria (tipo, diurna y/o nocturna, frecuencia de episodios de incontinencia)

1. Continua

2. Intermitente

a. Diurna (Relación con urgencia, ejercicio, risa)

b. Nocturna (enuresis) (momento de la noche)

III. Urgencia miccional

IV. Nicturia (frecuencia miccional nocturna)

B. Síntomas durante la fase de vaciado o miccional

I. Dificultad para iniciar la micción

II. Micción por prensa abdominal

III. Disminución del chorro miccional

IV. Micción intermitente o interrumpida (no continua)

\section{Otros síntomas}

I. Infección del tracto urinario (ITU)

II. Maniobras anti-pis (para posponer la micción o suprimir

la sensación de urgencia miccional)

III. Sensación de micción incompleta

IV. Disuria

V. Goteo Terminal

\section{- Antecedentes personales}

Historia de infecciones del tracto urinario (ITU)

Historia de RVU bilateral o unilateral cambiante de lado

Historia de retención urinaria

Tratamiento farmacológico previo relacionado con el tracto urinario (TU) o no

Cirugías previas

- Función anorrectal (historia de estreñimiento, encopresis)
- Antecedentes familiares de RVU y MTUI

Diario miccional (a partir de la edad de control miccional)

Registro diario en ambiente familiar y escolar de ingesta de líquidos, diuresis y defecación durante un mínimo de 48 horas. Se obtiene importante información acerca de frecuencia miccional, volumen total, medio y máximo orinado en 24 horas, distribución del volumen orinado de día y de noche, frecuencia y severidad de episodios de incontinencia diurna y nocturna, existencia de poliuria, frecuencia de nicturia, patrón y volumen de ingesta de líquidos, frecuencia de defecación y severidad de encopresis.

\section{Observación miccional durante 4 horas (en lactantes y pre- escolares)}

Test no invasivo de valoración de la función del TUI en los niños por debajo de la edad de control miccional. Consiste en la valoración de la capacidad de vaciamiento vesical mediante la observación continua del patrón miccional, el volumen de llenado y el residuo postmiccional, con ayuda de la ecografía y la medida de diuresis pesando el pañal.

\section{Exploración física}

General con especial atención en la exploración de las regiones:

- Genital: Vulva e introito vaginal en las niñas (sinequia vulvar, sinus urogenital). Pene y meato uretral en los niños (fimosis, estenosis meatal, hipospadias)

- Sacra: Integridad del sacro. Estigma cutáneo (disrafismo subyacente)

- Miembros inferiores: Deformidades o asimetrías.

- Abdominal: Impactación fecal

- Perineal y anal: Sensación perineal y perianal. Posición anal, tono esfínter anal y reflejo bulbocavernoso (la inervación de la región perineal y vésico-esfinteriana, procede de los segmentos sacros S1-S4)

\section{Analítica de orina \\ Despistaje de ITU. Presencia de bacteriuria asinto- mática.}

\section{Ecografía renal y vesical}

Evaluación anatómica del tracto urinario superior (TUS). Afectación funcional renal (disminución de cortical renal, diferenciación córtico-medular).

Evaluación anatómica del TUI. Medida del espesor de la pared vesical, aunque no hay parámetros de normalidad en la actualidad.

Valoración del RVU (eco-cistografía con galactosa)

Medida ecográfica del residuo postmiccional.

Signos indirectos o de sospecha de MTUI en base a los hallazgos de otras pruebas complementarias dentro del protocolo de estudio del RVU (Tabla I):

Cistouretrografía miccional seriada (CUMS):

- Presencia de RVU bilateral. Fase de inicio del RVU.

- Fase de llenado: Morfología vesical (especulación, vejiga pseudodivertícular); Volumen de llenado al que se produce el RVU. 
- Fase miccional: Presencia o mayor grado de RVU durante la micción (RVU activo); Morfología cuello vesical y uretral (dilatación proximal o "uretra en remolacha o peonza", impronta o estenosis funcional uretral; Reflujo vaginal).

- Residuo postmiccional.

Gammagrafía renal con ácido dimercapto - succínico (DMSA): Afectación de la función renal diferencial. Riñón pequeño (hipoplásico) o anulación funcional renal. Presencia, extensión y severidad de cicatrices renales.

\section{Flujometría con electromiografía (EMG) simultánea}

Es la técnica urodinámica menos invasiva y debe ser la mas frecuentemente utilizada en la evaluación inicial de la función TUI en la edad pediátrica, a partir de la edad de control miccional. Es la medida del volumen de orina evacuado por unidad de tiempo $(\mathrm{ml} / \mathrm{s})$. Aporta datos sobre la fase miccional. Consiste en la micción espontánea realizada en un flujómetro (que convierte el peso de la orina en valor de flujo urinario).

Se realiza con EMG simultánea, que consiste en la medida de la actividad electromiográfica del esfínter periuretral mediante la colocación de dos electrodos de contacto perianales. La actividad EMG del esfínter durante la micción es uno de los parámetros más importantes a valorar durante la flujometría en la edad pediátrica, de cara al diagnóstico urodinámico.

Sus resultados juegan un papel fundamental en la decisión sobre qué niños se beneficiarán del estudio urodinámico completo (cistometría) así como en el seguimiento urodinámico durante el tratamiento y la rehabilitación miccional. Por ello, es necesario repetir la prueba en dos o tres ocasiones, con el niño hidratado y con deseos de orinar, para asegurar un volumen miccional mínimo razonable y para extraer conclusiones en relación al diagnóstico urodinámico. Es más fiable a partir de los 4 años de edad y se requieren adaptaciones específicas para que la postura al orinar les permita un vaciamiento y una relajación adecuados.

Los parámetros a medir de mayor utilidad en la edad pediátrica son:

\section{VOLUMEN MICCIONAL}

No valorable si $<50 \%$ de la Capacidad Vesical Esperada según edad (CVE).

\section{PATRÓN O CONFIGURACIÓN CURVA FLUJOMÉTRICA}

Determinado por la contractilidad del detrusor, prensa abdominal y la resistencia uretral a la salida de la orina.

Una curva normal, independientemente del sexo y la edad, es una curva de flujo continuo y bordes suaves en forma de campana, con relajación de la actividad esfinteriana durante toda la micción. No obstante, el 1\% de los preescolares normales tienen curvas en meseta o flujo interrumpido. Por ello, es conveniente repetirla, al menos en dos ocasiones.
Los patrones de afectación más frecuentes en la edad pediátrica los describimos como:

- Curva en torre (sugiere hiperactividad del detrusor)

- Curva en meseta (sugiere obstrucción anatómica estática de la salidad de la vejiga)

- Curva irregular o en staccato (continua pero fluctuante) (sugiere micción no coordinada o disfunción miccional propiamente dicha)

- Curva interrumpida o fraccionada (sugiere hipoactividad del detrusor)

\section{EMG PERIANAL}

La micción normal ocurre en presencia de relajación pasiva del esfínter periuretral.

El aumento de la actividad EMG durante la micción puede significar una MICCIÓN NO COORDINADA o DISFUNCIONAL O DISFUNCIÓN MICCIONAL propiamente dicha, sobre todo si se asocia a residuo postmiccional significativo. Asimismo una MICCIÓN POR PRENSA puede producir un aumento de la actividad EMG durante la micción.

\section{RESÍDUO POSTMICCIONAL (Medida ecográfica)}

La medida del residuo postmiccional se realiza en la actualidad mediante ecografía a tiempo real.

A excepción de los lactantes pequeños, los niños pueden vaciar su vejiga en cada micción de forma efectiva. Incluso en los lactantes, durante la observación miccional de 4 horas, al menos una micción se sabe es completa y no deja residuo.

Interpretación:

- Debe medirse inmediatamente después de la micción (no después de 5 minutos de la finalización de la misma. Esto evita la medida de residuo falsamente positivo en presencia de reflujo vésico-ureteral de alto grado). Si este tiempo se excede al preparar el ecógrafo, se debe sustraer 1-2 ml por cada minuto de tiempo sobrepasado la partir de los 5 min.) en la medida del residuo.

- Su AUSENCIA es un dato importante de NORMALIDAD (Aunque no excluye la MTUI)

- Valorable clínicamente si es > $5 \mathrm{ml}$, a partir de $5 \mathrm{~min}$. de la finalización de la micción. Esta cifra es el valor más alto de residuo no asociado a ITU.

Entre $5-20 \mathrm{ml}$, el residuo es significativo y debe repetirse para confirmar el dato.

Un residuo $>20 \mathrm{ml}$ en más de una ocasión, indica un vaciamiento inefectivo incompleto o anormal, siempre que la vejiga no estuviera en exceso llena, respecto a lo que es normal para cada niño.

\section{Estudio URODINÁMICO (CISTOMETRÍA PEDIÁTRICA)}

Estudio de la función vesico-uretral en sus dos fases de llenado y vaciado. El escaso tiempo de transición entre ambas fases en la edad pediátrica (ya que los niños orinan a menudo sin permiso) hace que la prueba se realice en un único estudio durante el llenado y el vaciado vesical, al que denominamos cistometría pediátrica. La distinción entre cistometría de llenado y curva de presión/flujo (fase miccional) queda postpuesta para el análisis de las curvas. Se puede realizar por vía suprapúbica o transure- 
tral. Nosotros preferimos el abordaje transuretral de manera habitual por ser menos invasivo, aunque en el lactante pequeño tiene una gran utilidad la ruta suprapúbica por disminuir significativamente el efecto obstructivo del catéter uretral. Para minimizar los artefactos por la irritación de los catéteres se debe dar tiempo a que el niño se adapte al mismo. La repetición del llenado puede ser clave para una mejor interpretación del ciclo.

El llenado debe ser fisiológico, esto es, que no exceda la tasa máxima esperada de producción de orina por los riñones. (Peso en $\mathrm{Kg} / 4$ ). En la edad pediátrica se prefiere emplear el límite del 5-10\% de la CVE según edad, según la fórmula de Koff (Koff, 1983 (5) (Capacidad vesi$\mathrm{cal}=30$ (edad en años+2)) expresado en $\mathrm{ml} / \mathrm{min}$, siendo conveniente que no exceda de $10 \mathrm{ml} / \mathrm{min}$.

Si el equipo está disponible, la técnica ideal es el uso del llenado natural de la vejiga (o cistometría ambulatoria) que proporciona una velocidad verdadera de llenado fisiológico y datos mas precisos de la actividad vesical durante la actividad del niño durante el día y la noche.

La cistometría comienza con el llenado vesical con suero fisiológico a temperatura ambiente a una velocidad de llenado "fisiológica", con medida de los siguientes parámetros:

-Presión vesical (Pves), mediante sonda vesical de doble vía de $6 \mathrm{Fr}$ que se conecta a tranductor de presión intravesical y a línea de llenado.

-Presión abdominal (rectal) (Pabd), mediante sonda-balón rectal de $7 \mathrm{Fr}$ que se conecta a transductor de presión abdominal. Su misión es evitar la influencia de las maniobras de aumento de Pabd sobre la $P$ ves.

-Presión detrusor (Pdet = Pves - Pabd). Es la verdadera presión intrínseca vesical sin las influencias de la Pabd.

-EMG esfínter periuretral (mediante electrodos perianales de contacto)

La cistometría finaliza con el estudio de la relación entre la presión del detrusor y el flujo urinario durante la fase de vaciado vesical (curva de P/F)

\section{CISTOMETRÍA DURANTE EL LLENADO CON EMG \\ Parámetros a medir: \\ - Función del detrusor \\ - Sensación vesical \\ - Acomodación vesical \\ - Capacidad vesical \\ - Actividad electromiográfica esfinteriana simultánea}

Función del detrusor durante el llenado: Pdet ( $\mathrm{cm}$ de $\mathrm{H}_{2} \mathrm{O}$ ) Se considera que la FUNCION DEL DETRUSOR ES NORMAL cuando permite un llenado vesical con pequeños o sin cambios de presión (proceso denominado "acomodación"), a pesar del aumento del volumen de llenado vesical, sin la existencia de contracciones fásicas involuntarias, a pesar de su provocación (tos, Valsalva...).

\section{Contracciones involuntarias del detrusor (CID)}

La presencia de contracciones involuntarias del detrusor, espontáneas o provocadas, que se traducen en aumentos de la presión del detrusor por encima de $15 \mathrm{~cm}$ de $\mathrm{H}_{2} \mathrm{O}$ sobre la línea de base, con/sin sensación de deseo miccional o maniobras anti-pis, a menudo acompañadas de aumento de actividad EMG del esfínter, con o sin escape urinario, son característicos de la presencia de HIPERACTIVIDAD DEL DETRUSOR.

- Fásica (con CID durante el llenado, con o sin incontinencia) (Figura 1)

- Terminal (con escasas o solo una CID al final del llenado que provoca micción involuntaria) (Figura 2)

\section{Presión del detrusor de escape en reposo (PER) o Presión de punto de fuga (LPP) (ICS)}

Es la presión del detrusor a la cual se produce el primer escape de orina por uretra. Excluye cualquier aumento de presión abdominal asociado, pero incluye el aumento de actividad EMG para evitar la pérdida.

Su importancia radica en:

- Su valor pronóstico: Mayor incidencia de lesión del TUS cuando supera los $40 \mathrm{~cm}$ de $\mathrm{H}_{2} \mathrm{O}$ (vejiga de alto riesgo).

- Su valor en el tratamiento: Instauración del tipo de terapia miccional.

\section{Sensación de deseo miccional:}

De difícil valoración en la edad pediátrica. En el niño mayor podrá valorarse la primera sensación y/o ganas normales de orinar y sensación de urgencia miccional. En general, y sobre todo en el niño de menor edad, el parámetro que puede valorarse en la edad pediátrica es la presencia o ausencia-disminución del deseo miccional (como ocurre en el detrusor hipo o acontráctil)

\section{Compliance o acomodación vesical:}

Cambio de volumen para un determinado cambio de presión. Se obtiene dividiendo el incremento de volumen entre incremento de presión $\left(\mathrm{ml} / \mathrm{cm}\right.$ de $\left.\mathrm{H}_{2} \mathrm{O}\right)$. Varía en función de la velocidad de llenado, parte de la curva en la que se calcula, configuración y espesor de la vejiga, propiedades mecánicas de la pared vesical, propiedades de Contractilidad-Relajación del músculo detrusor.

En la edad pediátrica, la compliance varía en función de la capacidad esperada según edad, aumenta con la edad, y no hay valores de referencia de normalidad. Se consideran normales las presiones del detrusor de $\leq 10 \mathrm{~cm}$ de $\mathrm{H}_{2} \mathrm{O}$ a la capacidad esperada según edad. Un método de medida es el que considera que los valores de Compliance normales en el niño neurologicamente normal son: $C^{\prime} \geq 0,05 \times C V E$. Pero más importante que los valores numéricos es la configuración de la curva de presión durante el llenado. Cuando durante el llenado vesical (aumento del volumen) hay pocos cambios en la presión del detrusor (presiones bajas y seguras) la acomodación se considera normal. Su valor debe expresarse junto a la velocidad de infusión y la parte de la curva en la que se ha calculado.

Los valores que debemos medir de forma estandar según la ICS son:

- Compliance al inicio del llenado

- Compliance FINAL: El valor más importante es la acomodación al final del llenado a capacidad vesical máxima. 


\section{Capacidad vesical o Capacidad cistométrica Máxima:}

Volumen vesical fisiológico al cual el niño comienza a orinar con grandes deseos.

\section{Capacidad vesical esperada según edad (CVE):}

Es la capacidad teórica que debería tener el niño a determinada edad.

\section{Función uretral durante el llenado:}

Medida mediante el registro de la actividad electromiográfica del suelo pélvico usando los electrodos de contacto perianales.

\section{CURVA DE PRESIÓN-FLUJO (P/F) CON EMG}

- Estudia la fase miccional.

- Estudio de la relación entre la presión del detrusor y el flujo urinario durante la fase de vaciado vesical. Es la segunda parte de la cistometría y es básica para la confirmación del diagnóstico urodinámico.

- Una curva flujométrica alterada o un flujo miccional disminuido deben ser evaluados por la curva de P/F para determinar la causa de estas alteraciones.

- Se realiza poniendo al paciente a orinar, a máxima capacidad, con las sondas vesical y rectal y los electrodos de registro de la EMG simultánea.

- Durante la micción se produce un aumento de la presión del detrusor que depende de la función contráctil del detrusor, resistencia uretral y flujo urinario.

- La presión del detrusor es máxima cuando el flujo urinario es cero (contracción isovolumétrica).

- La presión del detrusor es mínima a máximo flujo (contracción isotónica) (Pdet a máximo flujo).

- Una micción normal se inicia por una contracción voluntaria del detrusor, que consigue el vaciamiento vesical completo.

Parámetros a medir:

- Configuración de la curva

- EMG esfínter periuretral

- Volumen miccional

- Función del detrusor durante la micción

Interpretación:

Permite diferenciar si la presión del detrusor durante la micción es debida a la contracción del detrusor, voluntaria o involuntaria (una contracción involuntaria del detrusor precede a la relajación esfinteriana) o a la prensa abdominal.

La presión del detrusor normal varía entre:

$50-80 \mathrm{~cm}$ de $\mathrm{H} 2 \mathrm{O}$ en los varones (66)

$35-65 \mathrm{~cm}$ de $\mathrm{H} 2 \mathrm{O}$ en las niñas (57)

- Una presión elevada del detrusor durante la micción puede indicar hiperactividad del detrusor:

- Secundaria a:

Micción no coordinada (MNC) (obstrucción funcional del TUI): suele ir asociada a residuo postmiccional elevado (curva irregular o en staccato)

va en mesetal

Obstrucción orgánica (estenosis uretral, VUP) (cur-

En ambos casos la presión del detrusor es alta y el flujo bajo.
- Primaria (contracción involuntaria del detrusor) con MNC secundaria, que no suele ir asociada a residuo postmiccional (curva en torre)

- Una presión baja del detrusor durante la micción (asociada a flujo bajol que prolonga el vaciado o falla al conseguir un vaciamiento efectivo, es compatible con HIPOACTIVIDAD DEL DETRUSOR (curva interrumpida o fraccionada secundaria a micción con ayuda de prensa abdominal (antigua vejiga perezosa), con/sin MNC secundaria por prensa, a menudo con residuo postmiccional. En el DETRUSOR ACONTRÁCTIL no se demuestra contracción del detrusor durante el estudio.

Presión pre-miccional del detrusor: Presión del detrusor inmediatamente antes de la contracción isovolumétrica inicial. Su elevación es importante en el diagnóstico de hiperactividad terminal del detrusor.

Actividad del esfínter durante la micción: La valoración de la relajación/contracción del esfínter durante la fase miccional se realiza mediante el registro de la actividad EMG del esfínter a través de los electrodos de contacto perianales. Esta medida proporcionaría el diagnóstico de coordinación o descoordinación del esfínter durante la micción.

DISFUNCIÓN MICCIONAL: Diagnóstico urodinámico caracterizado por un flujo intermitente o fluctuante (curva en en staccato) debido al aumento intermitente involuntario de la actividad del esfínter uretral estriado externo o suelo pélvico durante la micción, y que deja residuo postmiccional. Es importante valorar si la relajación del esfínter precede o no a la contracción del detrusor. En ocasiones una contracción involuntaria del detrusor precede a la relajación del esfínter abriéndolo de forma involuntaria.

La valoración de la actividad EMG del esfínter en la curva miccional de $P / F$, será necesaria para hacer el diagnóstico diferencial entre micción disfuncional y micción por prensa; en esta última, el aumento de la actividad esfinteriana es voluntario y se debe al incremento de la prensa abdominal.

\section{Resíduo postmiccional (R)}

El Residuo postmiccional -volumen de orina que queda en la vejiga tras la micción- es mucho más valorable tras la flujometría libre. La presencia de residuo tras la curva de $P / F$, en ausencia del mismo tras la flujometría libre, debe ser considerado como artefacto por las circunstancias en las que se realiza el test.

\section{PATRONES URODINÁMICOS}

Los hallazgos urodinámicos pueden clasificarse en 4 patrones básicos de alteración funcional; dos que afectan de forma primaria a la fase de llenado vesical (patrones 1 y 2) (Figuras 1 y 2) y dos que afectan de forma primaria a la fase de vaciado vesical o fase miccional (patrones 3 y 4), aunque con frecuencia, ambas formas de alteración se presentan conjuntamente (Figuras 3 y 4 ).

a. Alteraciones de la fase de llenado Patrón 1: Hiperactividad del detrusor fásica 


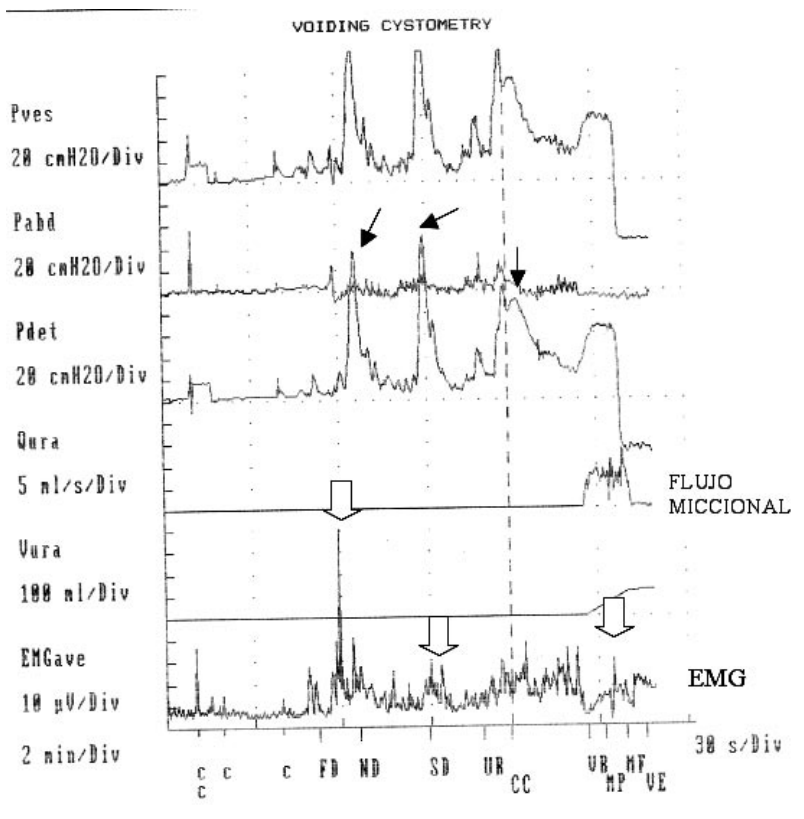

FIGURA 1. PATRON 1: HIPERACTIVIDAD DEL DETRUSOR TIPO I (FASICA) con contracciones involuntarias del detrusro (CID) (flechas negras) durante el llenado, con aumento de la actividad electromiográfica (EMG) (flechas blancas) durante las CID del llenado y en la mitad de la micción (Micción no coordinada secundaria). NO RESIDUO POSTMICCIONAL.

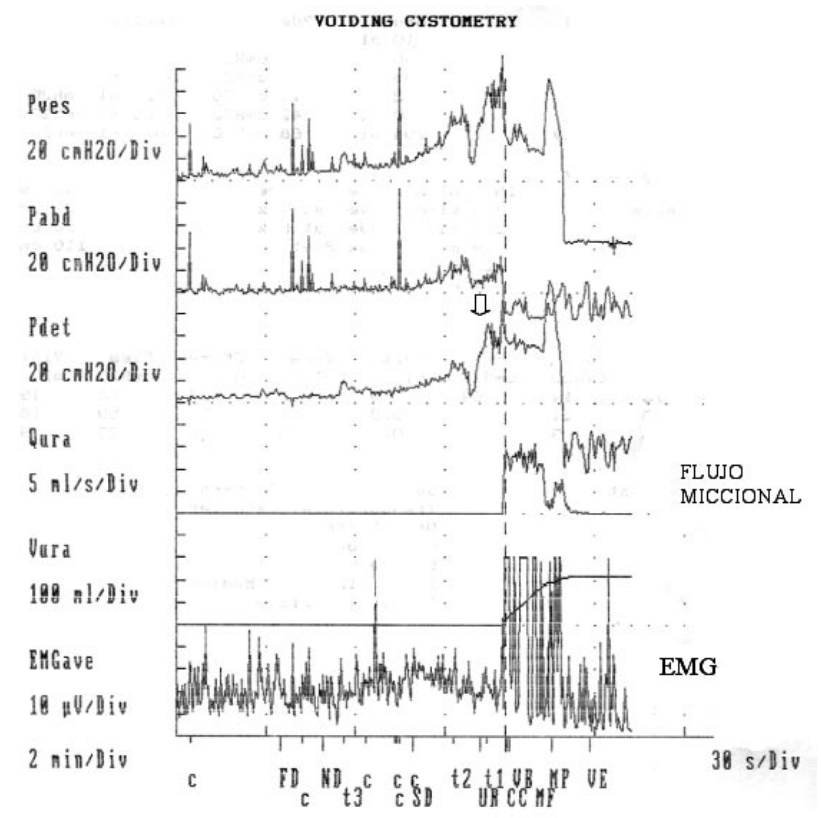

FIGURA 2. PATRON 2: HIPERACTIVIDAD DEL DETRUSOR TERMINAL . Contracción involuntaria del detrusor premiccional (flecha). Asociación a MICCION DISFUNCIONAL (irregular o en staccato, bifásicol con aumento de la actividad EMG durante la micción y RESIDUO POSTMICCIONAL.

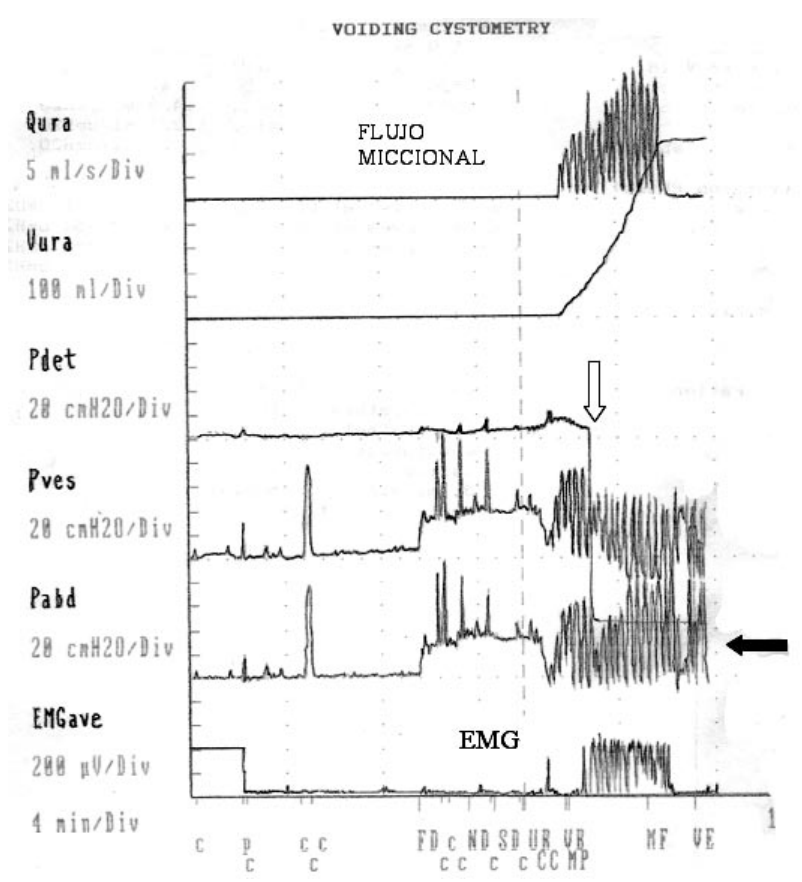

FIGURA 3. PATRON 3: HIPOACTIVIDAD DEL DETRUSOR / $\mathrm{sa}^{-}$ lida de sonda por esfuerzo vigoroso al orinar -flecha blanca-) CON MICCION NO COORDINADA POR PRENSA (flecha negra) ( $\neq$ EMG durante la micción) (patrón de flujo miccional interrumpido o intermitente). RESIDUO POSTMICCIONAL ASOCIADO.

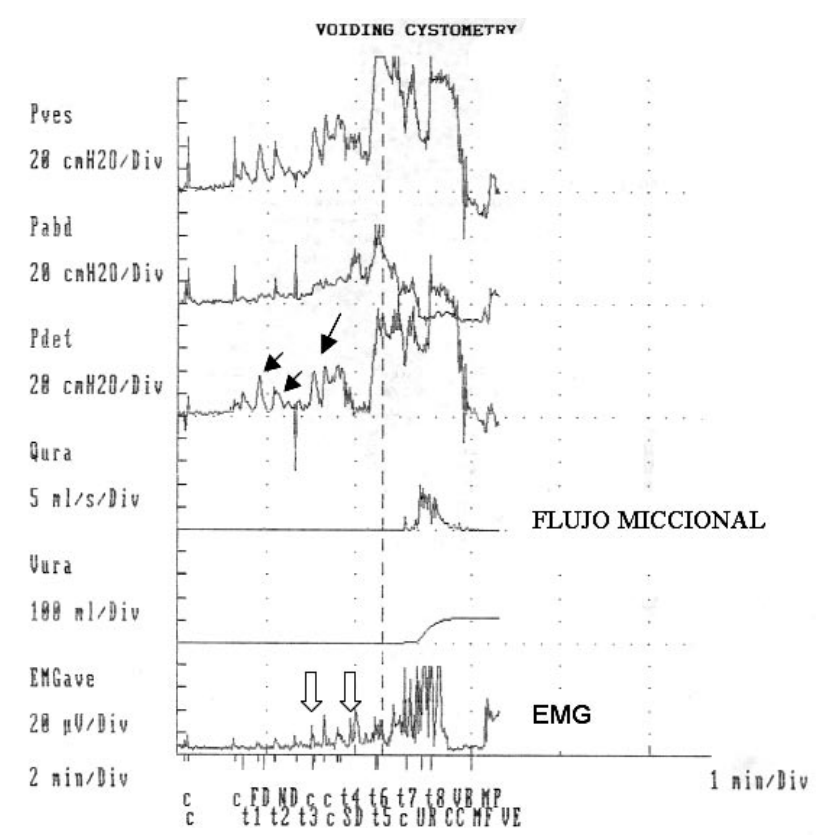

FIGURA 4. PATRON 4: MICCION DISFUNCIONAL IFlujO miccional bifásico, irregular, en staccatol CON HIPERACTIVIDAD DEL DETRUSOR FASICA /CID durante el llenado -flechas negras-, con aumento de la actividad EMG -flechas blancas-). RESIDUO POSTMICCIONAL. 
Patrón 2: Hiperactividad del detrusor terminal

b. Alteraciones de la fase miccional

Patrón 3: Hipoactividad vesical

Patrón 4: Disfunción miccional, Micción disfuncional o Micción no coordinada

c. Combinación de alteraciones de la fase de llenado y miccional

Patrón 1) HIPERACTIVIDAD DEL DETRUSOR TIPO I o FÁSICA: con contracciones involuntarias del detrusor (CID) según la ICCS- (o contracciones no inhibidas del detrusor), compliance vesical final normal o disminuida, Presión de punto de fuga del detrusor (LPP) -según la ICS- o PER (Pdet de escape en reposo, en ausencia contracción o elevación de la Pabd) elevada, reducida capacidad cistomanometrica, presiones pre-miccionales y miccionales normales o elevadas y asociación a micción no coordinada secundaria, que no suele dejar residuo postmiccional.

Patrón 2) HIPERACTIVIDAD DEL DETRUSOR TIPO II o TERMINAL: con CID ausentes o de baja frecuencia e intensidad, compliance final disminuida, Capacidad cistomanometrica aceptable, y presiones pre y miccionales elevadas.

Patrón 3) HIPOACTIVIDAD DEL DETRUSOR (antigua vejiga "Perezosa") con Pdet baja durante la micción, flujo miccional bajo, micción no coordinada secundaria por prensa abdominal y residuo postmiccional con frecuencia elevado o ausente.

Patrón 4) DISFUNCIÓN MICCIONAL PROPIAMENTE DICHA O MICCIÓN DISFUNCIONAL (ICCS) (MICCIÓN NO COORDINADA PRIMARIA), con residuo postmiccional elevado, en frecuente asociación con hiperactividad del detrusor -con mayor frecuencia de tipo terminal-

\section{TRATAMIENTO URODINÁMICO DE LA MALFUNCIÓN DEL TRACTO URINARIO INFERIOR}

\section{OBJETIVOS}

\section{A). VACIAMIENTO VESICAL EFECTIVO}

I. REHABILITACIÓN DEL TRACTO URINARIO INFERIOR TERAPIA MICCIONAL) BAJO CONTROL VOLUNTARIO: MODIFICACIÓN DEL COMPORTAMIENTO DEL NIÑO

\section{Biofeedback:}

a. Relajación esfinteriana (visualización del registro EMG en relajación y contracción suelo pélvico en forma de registro o curva o imagen con/sin sonido)

b. Flujo urinario (observación de la curva flujométrica durante la micción)

\section{Reeducación miccional}

a. Micción programada e ingesta líquida adaptada individualmente

b. Micción doble (o triple)

c. Aprendizaje y adaptación postural durante la micción (optimización de la relajación del suelo pélvico)

d. Hacer entender función vesical y en que medi- da el niño se separa de la normalidad, para involucrar y motivar a los padres y al niño

\section{CATETERIZACIÓN VESICAL INTERMITENTE (CVI)}

\section{B). PREVENCIÓN DE ITU}

I. PROFILAXIS ANTIBIÓTICA nocturna continua o discontinua con antisépticos urinarios

C). RELAJACIÓN MÚSCULO DETRUSOR: NORMALIZACIÓN DE PRESIONES INTRAVESICALES (disminución Pdet, evitar CNI, mejoría de la acomodación vesical)

\section{ANTICOLINERGICOS: OXIBUTININA oral}

Dosificación de comienzo a dosis muy bajas $(0,2 \mathrm{mg} / \mathrm{Kg} /$ día) con aumento progresivo de la dosis hasta efecto terapéutico deseado (minimiza efectos secundarios transitorios)

Antes del tratamiento con anticolinérgicos se debe asegurar un aprendizaje o procurar un vaciamiento vesical efectivo (según las medidas antes comentadas)

D). SUSTITUCIÓN DE LA TERAPIA MICCIONAL DURANTE LA NOCHE. DISMINUCIÓN DE LA PRODUCCIÓN DE ORINA NOCTURNA

I. DESMOPRESINA oral (liofilizado o comp) o nasal La asociación de desmopresina y oxibutinina se emplea con efectividad en los casos de persistencia clínica de la enuresis nocturna tras el tratamiento con oxibutinina, a pesar de la resolución diurna de los síntomas, no infrecuente en los niños con patrones urodinámicos de hiperactividad del detrusor tipo II (de baja compliance vesical y elevada presión premicional, a pesar de que la capacidad vesical efectiva sea aceptable)

\section{TRATAMIENTO SEGÚN PATRÓN URODINÁMICO}

PATRON 1: HIPERACTIVIDAD DEL DETRUSOR FÁSICA O TIPO I

\section{Rehabilitación TUI}

- Reeducación miccional

Micción programada

Micción doble

- CVI en el lactante o preescolar con vejiga de alto riesgo

Tratamiento farmacológico

- Anticolinérgicos: Oxibutinina: 0,2-0,5 mg/Kg/día

- Profilaxis antibiótica nocturna

PATRÓN 2: HIPERACTIVIDAD DEL DETRUSOR TERMINAL O TIPO ॥

\section{Rehabilitación TUI}

- Reeducación miccional

Tratamiento farmacológico combinado según el caso - Anticolinérgicos: Oxibutinina: 0,2-0,5 mg/Kg/día - Desmopresina oral: 1-2 comp liofilizados/día por la noche (sustituye a la terapia miccional por la noche) si persisten síntomas nocturnos (enuresis)

- Profilaxis antibiótica nocturna 


\section{PATRON 3: HIPOACTIVIDAD DEL DETRUSOR}

Tratamiento farmacológico

- Profilaxis antibiótica nocturna

\section{Rehabilitación TUI}

- Reeducación miccional

- CVI, en casos refractarios o en el lactante

\section{DISFUNCIÓN MICCIONAL O MICCIÓN NO COORDINADA}

\section{Rehabilitación TUI}

- Biofeedback

- Reeducación miccional

\section{Tratamiento farmacológico}

- Anticolinérgicos: Oxibutinina: 0,2-0,5 mg/Kg/día (si hiperactividad del detrusor significativa asociada

- Profilaxis antibiótica nocturna

\section{ESTUDIO PROSPECTIVO DE 63 PACIENTES PEDIÁ- TRICOS PORTADORES DE RVU PRIMARIO}

\section{MATERIAL Y MÉTODOS}

Estudio prospectivo y consecutivo de 92 pacientes pediátricos portadores de Reflujo Vésico-Ureteral (RVU), reclutados a lo largo de un año (Julio2004-Junio 2005), 63 de los cuales corresponden al diagnóstico de RVU primario y que constituyen nuestra población de estudio.

\section{ANTECEDENTES DE LOS PACIENTES}

Antes del reclutamiento, estos 63 pacientes fueron tratados mediante tratamiento médico conservador con profilaxis antibiótica nocturna continua, hasta la edad de los 8 años, SIN tratamiento urológico PREVIO, entendiendo por dicho tratamiento: 1) la intervención quirúrgica sobre la unión uretero-vesical (ureteroneocistostomía o antirreflujo endoscópico) u otra cirugía previa del tracto urinario, y/o 2) la intervención sobre la posible Malfunción del TUI (MTUI) subyacente. El seguimiento de estos pacientes fue realizado mediante controles clínicos con medida periódica de la presión arterial, analíticos (sangre y orina), ecográficos, cistográficos (mediante cistouretrografía miccional seriada -CUMS- y/o ecocistografía miccional con galactosa), urográficos y gammagráficos (mediante Acido Di-Mercapto-Succínico-DMSA-).

\section{CRITERIOS DE INCLUSIÓN}

Pacientes pediátricos (edad $\leq 15$ años)

RVU primario en el momento del reclutamiento

\section{CRITERIOS DE EXCLUSIÓN}

Se han descartado del estudio, por definición, 29 casos de RVU compatibles con "Síndrome de válvulas de uretra posterior like" (VUPL) (RVU fetal bilateral de alto grado con insuficiencia renal), así como los casos de RVU secundario a malformaciones anatómicas estructurales (duplicación renal completa, riñón en herradura, ureterocele, divertículo vesical, VUP, extrofia, pseudoextrofia vesical), malformaciones anorrectales o afectación neurológica (vejiga neurógena).

\section{CARACTERISTICAS DE LA POBLACIÓN}

El estudio comprende una población pediátrica de 63 pacientes portadores de RVU primario grado IIVV, 28 niñas y 35 varones, de edades comprendidas entre 6 meses y 15 años de edad, con una edad media de 8 años en las niñas (rango entre 2-15 años) y de 4,2 años (rango entre 6 meses y 15 años) en los varones.

\section{PROTOCOLO DE ESTUDIO}

Se ha llevado a cabo una evaluación del RVU utilizando un protocolo de estudio estandarizado consistente en la realización de: analítica de orina, ecografía renal y vesical, CUMS, gammagrafía renal con DMSA y estudio de la función del TUl mediante historia miccional y de hábito fecal, diario miccional, estudio urodinámico no invasivo de la función del TUI (diario miccional, flujometría con electromiografía, observación miccional durante 4 horas) y estudio cistométrico durante las fases de llenado y vaciado del ciclo miccional (Tabla I).

\section{VARIABLES ESTUDIADAS}

Las variables estudiadas al reclutamiento han sido: sexo, edad, lateralidad y grado de RVU, función renal y función del TUI.

\section{GRADO DE REFLUJO}

El grado de RVU se ha calculado según el Sistema Internacional de clasificación radiológica del RVU (Lebowitz, 1985) (6). En los pacientes con RVU bilateral el lado de mayor grado de afectación es el que se ha empleado para comparaciones estadísticas y representación en las tablas.

\section{FUNCIÓN RENAL}

La función renal se ha considerado como normal o alterada en función de los hallazgos de la ecografía renal y la gammagrafia con DMSA (Tabla I), en base a la presencia y severidad de las cicatrices, y la función renal diferencial, considerando que existe daño renal cuando existe lesión focal o generalizada o función renal diferencial (FRD) menor o igual al $40 \%$.

\section{FUNCIÓN DEL TRACTO URINARIO INFERIOR}

La función del TUI se ha clasificado como normal o alterada en base a la presencia de síntomas y signos sugestivos de MTUI, y a los resultados del estudio urodinámico. La nomenclatura empleada está conforme a los estándares recomendados por la Sociedad de Contiencia Pediátrica Internacional (ICCS) (Neveus, 2006) (7). Los resultados del estudio urodinámico se han valorado en relación a las variables: edad, sexo, sintomatología urinaria, y antecedente de infección del tracto urinario inferior (ITU).

Se ha realizado flujometría+EMG en 52 pacientes $(83 \%), 28$ niñas y 24 varones, a partir de los tres años de edad. La cistometría se ha realizado en el $100 \%$ de los pacientes (63), como parte de los objetivos de estudio. La metodología del estudio urodinámico se ha aplicado conforme a los estándares recomendados por la ICCS y la ICS.).

\section{ANALISIS ESTADÍSTICO}

Se ha realizado mediante la chi cuadrado de Pearson y la corrección de Yates para tablas de contingencia. La asocia- 
TABLA I. PROTOCOLO DE ESTUDIO EN EL PACIENTE CON REFLUJO VESICO-URETERAL (RVU) PRIMARIO.

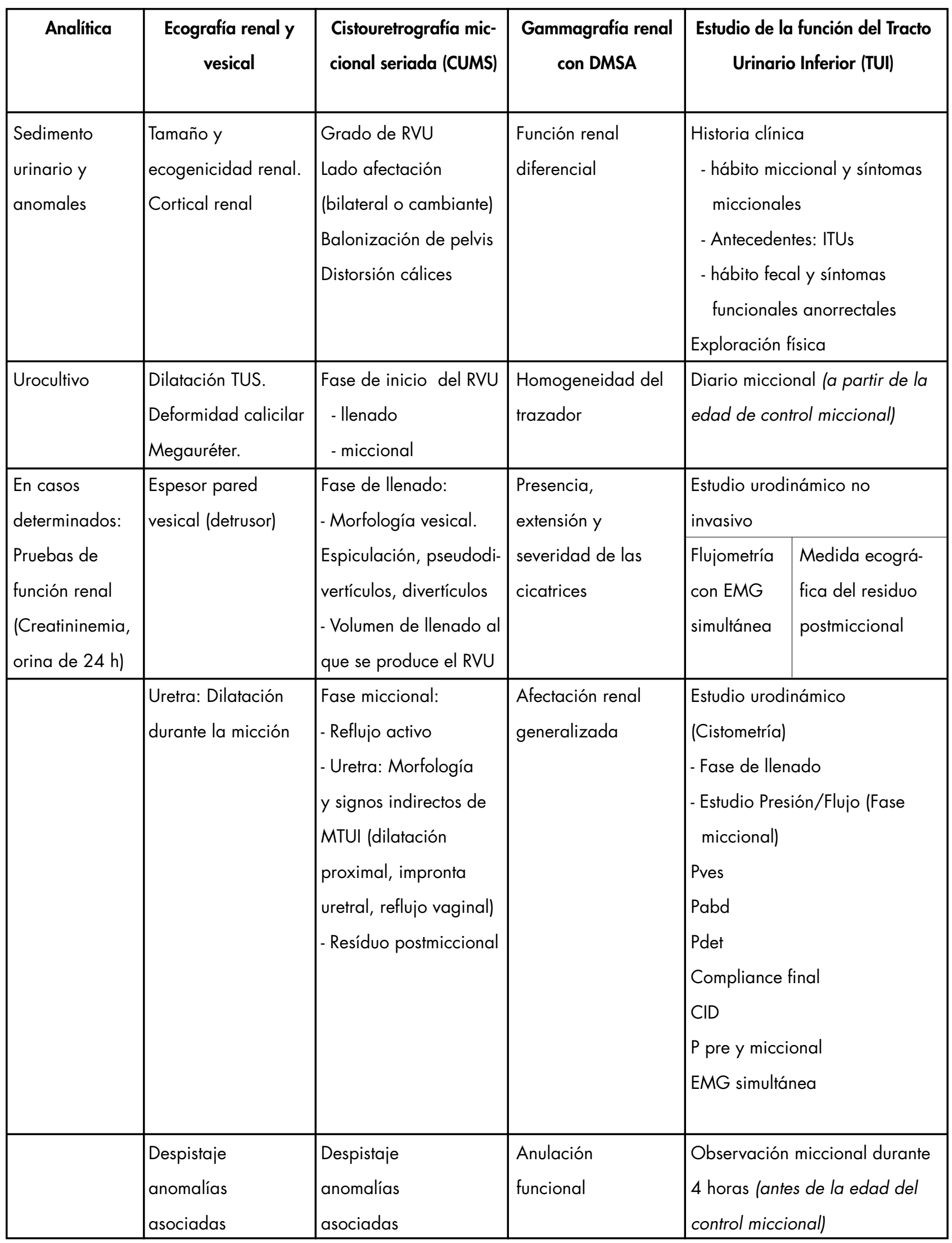

ITUs: Infecciones de tracto urinario inferior; TUS: Tracto urinario superior; EMG: Electromiografía; MTUI: Malfunción del tracto urinario inferior; CID: Contracciones involuntarias del detrusor. 
ción entre variables con $\mathrm{p}<0.05$ se ha considerado como estadísticamente significativa.

\section{RESULTADOS}

\section{LADO DE AFECTACIÓN Y GRADO DEL RVU, EDAD Y SEXO}

Lateralidad del RVU: El RVU es unilateral en 41 pacientes (18 niñas y 23 varones), con afectación del lado izquierdo en 24 pacientes ( 8 niñas y 16 varones) y del lado derecho en 17 pacientes (10 niñas y 7 varones) (tabla II). La afectación es bilateral en 22 pacientes (35\%) $(10$ niñas y 12 varones) lo que supone un total de 85 unidades renales (UR) en la serie presentada. Hay una distribución homogénea en la tasa de bilateralidad en relación al sexo, con una mayor frecuencia de afectación del lado izquierdo en el RVU unilateral en los varones $(70 \%)$, respecto a las niñas $(44 \%)$.

La edad media global de la población estudiada es de 5,9 años (rango entre 6 meses y 15 años), en la que los pacientes mayores de 5 años de edad (escolares, pre y adolescentes) suponen un $68 \%$ del total. En la distribución por sexos, es en la población femenina donde la edad media al reclutamiento es mas elevada (7,9 años), tanto en el RVU unilateral (7,7 años) como bilateral (8,2 años), respecto a os varones (edad media de 4,3 años). Los lactantes incluídos en el estudio son todos varones y suponen un $17 \%$ de la población estudiada (Tabla II).

Grado de RVU (Tabla III A y B): En el momento del reclutamiento, la gradación del RVU en la población estudiada corresponde a: grado II (7 pacientes, 9 UR), grado III (17 pacientes, 21 UR), grado IV (27 pacientes, 36 UR) y grado V (10 pacientes, 17 UR). Si consideramos como RVU de bajo grado los grados I-II, RVU de grado intermedio el grado III y RVU de alto grado los grados IV-V, hay una predominancia de RVU de alto grado al diagnóstico del $59 \%$ en la población estudiada. La distribución del grado de RVU por sexos muestra una mayor incidencia de RVU de alto grado $(69 \%)$ en la población de varones con respecto a las niñas $(54 \%)$, pero sin diferencias estadísticamente significativas, así como una mayor proporción de RVU de bajo grado en las niñas (18\%), respecto a los varones $(6 \%)$, con una distribución homogénea del RVU intermedio en ambos sexos (29\% en niñas versus $27 \%$ en varones). El RVU grado $\mathrm{V}$ es casi exclusivo de la población de varones ( $26 \%$ en varones versus $3,6 \%$ en niñas).

\section{DAÑO RENAL}

La función renal al diagnóstico se ha definido como alterada (daño renal) en el $52 \%$ de los pacientes (33 pacientes), con una mayor incidencia de daño renal entre los varones (71\%; $25 / 35)$, respecto a las niñas $(29 \%$; $8 / 28)$, SIN diferencias estadísticamente significativas.

La afectación renal está directamente relacionada con el mayor grado de RVU, no existiendo daño renal en los RVU de bajo grado. La existencia de daño renal está relacionada con la existencia de RVU de alto grado (IV-V) en el $62 \%$ de la población total (39/63); Por sexos, existe daño renal en un $53 \%$ de las niñas $(8 / 15)$ y en un $92 \%$ de los varones (22/24) con RVU de alto grado. Esta relación entre grado de RVU y daño renal es estadísticamente significativa con $\mathrm{p}<0,001$ (Tabla IV).

No existe asociación significativa entre la existencia de daño renal y la bilateralidad del RVU.

La afectación renal resulta ser generalizada, con $\mathrm{FRD}<40 \%$, en el $80 \%$ de los varones con daño renal $(20 / 25)$; un varón con anulación funcional renal), y de un $50 \%$ en las niñas $14 / 8$; una paciente con anulación funcional renal), correspondiendo el resto a lesiones focales cicatriciales, sin reducción de la FRD de forma global.

\section{MALFUNCIÓN DEL TUI RESULTADOS DEL ESTUDIO DE LA FUNCION DEL TUI}

1. Estudio no invasivo de la función TUI

Población femenina:

Presencia de síntomas de MTUI en el 96\% (27/28) de las niñas, 3 en edad preescolar, 16 escolar y 4 adolescentes. Antecedente de ITU en el $96 \%(27 / 28)$ de las niñas, con recurrencias durante la evolución en el $46 \%(13 / 28) ; 92 \%$ de ellas (12/13) con RVU de alto grado, $85 \%$ de ellas

TABLA II. RELACIÓN ENTRE LADO DE AFECTACIÓN DEL RVU, EL SEXO Y LA EDAD MEDIA AL DIAGNÓSTICO.

\begin{tabular}{|c|c|c|c|c|c|c|c|}
\hline SEXO & \multicolumn{3}{|c|}{ NIÑAS (total 28) } & \multicolumn{3}{|c|}{ VARONES (total 35) } & TOTAL \\
\hline \multirow[t]{2}{*}{ RVU } & \multicolumn{2}{|c|}{ UNILATERAL } & \multirow[t]{2}{*}{ BILATERAL } & \multicolumn{2}{|l|}{ UNILATERAL } & \multirow[t]{2}{*}{ BILATERAL } & \\
\hline & DERECHO & IZQUIERDO & & DERECHO & IZQUIERDO & & \\
\hline \multirow[t]{2}{*}{ № PACIENTES } & \multicolumn{2}{|c|}{18} & 10 & \multicolumn{2}{|c|}{23} & 12 & \\
\hline & 10 & 8 & & 7 & 16 & & \\
\hline EDAD MEDIA (años) & \multicolumn{2}{|c|}{7,7} & 8,2 & \multicolumn{2}{|c|}{2,2} & 3,16 & \\
\hline Edad media global (años) & \multicolumn{3}{|c|}{7,9} & \multicolumn{3}{|c|}{4,3} & 5,9 \\
\hline
\end{tabular}


TABLA III. NÚMERO DE PACIENTES SEGÚN GRADO DE REFLUJO, EN RELACIÓN CON EL DAÑO RENAL Y LA PRESENCIA DE MTUI. MTUI: MALFUNCIÓN DEL TRACTO URINARIO INFERIOR

III A: REFLUJO VESICO-URETERAL (RVU) EN LAS NIÑAS: 28 PACIENTES (P).

RVU UNILATERAL (18 p)

\begin{tabular}{|c|c|c|c|c|c|c|c|c|}
\hline \multicolumn{2}{|c|}{ GRADO DE RVU } & & & DAÑ & ENAL & MALFI & CION TUI & ANTECED RVU BILAT \\
\hline \multicolumn{2}{|l|}{ ॥ } & \multicolumn{2}{|l|}{3} & \multicolumn{2}{|l|}{$0 / 0$} & \multicolumn{2}{|l|}{$3 / 3$} & 1 \\
\hline \multicolumn{2}{|l|}{ III } & \multicolumn{2}{|l|}{5} & \multicolumn{2}{|l|}{$0 / 0$} & \multicolumn{2}{|l|}{$5 / 5$} & 3 \\
\hline \multicolumn{2}{|l|}{ IV } & \multicolumn{2}{|l|}{10} & \multicolumn{2}{|c|}{$4 / 10$} & \multicolumn{2}{|l|}{$9 / 10$} & 1 \\
\hline \multicolumn{2}{|l|}{ V } & \multicolumn{2}{|l|}{-} & & & & & \\
\hline TOTAL & & 18 & & \multicolumn{2}{|c|}{$4 / 18$} & \multicolumn{2}{|c|}{$17 / 18$} & $5 / 18$ \\
\hline \multicolumn{9}{|c|}{ RVU BILATERAL (10 p) } \\
\hline \multicolumn{4}{|c|}{ GRADO } & \multicolumn{2}{|c|}{ DAÑO RENAL } & \multicolumn{2}{|c|}{ MALFUNCION TUI } & \\
\hline \multicolumn{2}{|l|}{ ॥ } & \multicolumn{2}{|l|}{2} & \multicolumn{2}{|l|}{$0 / 2$} & \multicolumn{2}{|l|}{$2 / 2$} & \\
\hline \multirow[t]{2}{*}{ III } & |I-III & 1 & \multirow[t]{2}{*}{3} & \multirow{2}{*}{\multicolumn{2}{|c|}{$0 / 3$}} & $1 / 1$ & $3 / 3$ & \\
\hline & $\|I I-I\|$ & 2 & & & & $2 / 2$ & & \\
\hline \multirow[t]{2}{*}{ IV } & III-IV & 2 & \multirow[t]{2}{*}{4} & $1 / 2$ & $3 / 4$ & \multirow{2}{*}{\multicolumn{2}{|c|}{$4 / 4$}} & \\
\hline & IV-IV & 2 & & $2 / 2$ & & & & \\
\hline V & IV-V & 1 & & \multicolumn{2}{|l|}{$1 / 1$} & \multicolumn{2}{|l|}{$1 / 1$} & \\
\hline TOTAL & & 10 & & \multicolumn{2}{|c|}{$4 / 10$} & \multicolumn{2}{|c|}{$10 / 10$} & \\
\hline
\end{tabular}

TABLA III. NÚMERO DE PACIENTES SEGÚN GRADO DE REFLUJO, EN RELACIÓN CON EL DAÑO RENAL Y LA PRESENCIA DE MTUI. MTUI: MALFUNCIÓN DEL TRACTO URINARIO INFERIOR

III B. REFLUJO VESICO-URETERAL (RVU) EN LOS VARONES: 35 PACIENTES (P).

RVU UNILATERAL (23 p)

\begin{tabular}{|c|c|c|c|c|c|c|c|}
\hline \multicolumn{2}{|c|}{ GRADO DE RVU } & & & \multicolumn{2}{|c|}{ DAÑO RENAL } & MALFUNCION TUI & ANTECED RVU BILAT \\
\hline \multicolumn{2}{|l|}{$\|$} & \multicolumn{2}{|l|}{2} & \multicolumn{2}{|l|}{$0 / 2$} & $1 / 2$ & \\
\hline \multicolumn{2}{|l|}{ III } & \multicolumn{2}{|l|}{8} & \multicolumn{2}{|l|}{$3 / 8$} & $3 / 8$ & 1 \\
\hline \multicolumn{2}{|l|}{ IV } & \multicolumn{2}{|l|}{9} & \multicolumn{2}{|l|}{$8 / 9$} & $8 / 9$ & \\
\hline \multicolumn{2}{|l|}{ V } & \multicolumn{2}{|l|}{4} & \multicolumn{2}{|l|}{$4 / 4$} & $4 / 4$ & \\
\hline \multicolumn{2}{|l|}{ TOTAL } & \multicolumn{2}{|l|}{23} & \multicolumn{2}{|c|}{$15 / 23$} & $16 / 23$ & $1 / 23$ \\
\hline \multicolumn{8}{|c|}{ RVU BILATERAL (10 p) } \\
\hline \multicolumn{4}{|c|}{ GRADO } & \multicolumn{2}{|c|}{ DAÑO RENAL } & MALFUNCION TUI & \\
\hline \multicolumn{2}{|l|}{ ॥ } & \multicolumn{2}{|l|}{-} & & & & \\
\hline III & $\|-I\|$ & 1 & & \multicolumn{2}{|l|}{$0 / 1$} & $1 / 1$ & \\
\hline \multirow[t]{2}{*}{ IV } & III-IV & 3 & \multirow[t]{2}{*}{6} & $3 / 3$ & \multirow[t]{2}{*}{$5 / 6$} & \multirow[t]{2}{*}{$5 / 6$} & \\
\hline & IV-IV & 3 & & $2 / 3$ & & & \\
\hline V & IV-V & 5 & & \multicolumn{2}{|l|}{$5 / 5$} & $5 / 5$ & \\
\hline TOTAL & & 12 & & \multicolumn{2}{|c|}{$10 / 12$} & $11 / 12$ & \\
\hline
\end{tabular}


(11/13) a partir del segundo año de vida. Existe asociación significativa entre la presencia de ITU y MTUI en el grupo de niñas.

Flujometría con EMG realizada en el 100\% de las niñas (28) (a partir de los 3 años de vida): Morfología de la curva: normal o en campana en $2 / 28$, y anormal en el $93 \%$ de las niñas estudiadas (26/28); patrones de afectación: irregular o en staccato en el $54 \%(15 / 28)$, interrumpida polifásica en $8 / 28$, en torre en $3 / 28$. Actividad EMG: Aumento de la contracción del esfínter durante la micción presente en el $82 \%$ de las niñas (23/28). Residuo postmiccional presente en $22 / 28(79 \%)$.

\section{Población masculina:}

Presencia de síntomas de MTUI en el $51 \%$ (18/35) de los varones, 4 en edad preescolar, 11 escolar y 3 adolescentes. Antecedente de ITU en el $43 \%$ (15/35) de los varones, con recurrencias durante la evolución en el $23 \%(8 / 35)$; $100 \%$ de ellas (8/8) con RVU de alto grado, $75 \%$ de ellas $(6 / 8)$ en los primeros dos años de vida.

Flujometría con EMG realizada en el $69 \%$ de los varones (24/35) (a partir de los 3 años de vida): Morfología de la curva: Normal en campana en el $42 \%$ (10/24), y anormal en el 58\% (14/24); patrones de afectación: en torre en el $33 \%(8 / 24)$, interrumpida polifásica en $4 / 24$ y aplanada o en meseta en 2/24. Actividad EMG: Aumento de la contracción del esfínter durante la micción presente en el $42 \%$ de los varones (10/24), con residuo postmiccional asociado.

2. Estudio urodinámico (función de llenado y vaciado vesical)

Población femenina. Patrones de alteración urodinámica: - Disfunción miccional propiamente dicha o micción no coordinada primaria en el $64 \%$ de las niñas (18/28), con hiperactividad del detrusor asociada en el $78 \%$ de las mismas (14/18), de tipo terminal en 10/14 y fásica en 4/14 y residuo postmiccional significativo.

- Hipoactividad del detrusor con micción no coordinada secundaria por prensa abdominal en el $21 \%(6 / 28)$, con residuo postmiccional significativo en 4 .

- Hiperactividad del detrusor fásica en el $11 \%(3 / 28)$, sin residuo postmiccional.

Población masculina. Patrones de alteración urodinámica: -Hiperactividad del detrusor en el $74 \%$ de los varones (20/27), 11 de tipo fásico y 9 de tipo terminal, con micción no coordinada secundaria en el $40 \%$ (8/20), con residuo postmiccional significativo en 4 .

-Hipoactividad del detrusor en el $26 \%$ de los varones (7/27) con micción no coordinada secundaria, con residuo postmiccional significativo en 6 .

-De los 11 lactantes estudiados, todos varones sin insuficiencia renal (Creatininemia normal), en 2 se observa función normal de TUl (aunque con presiones premiccionales elevadas) sin residuo postmiccional y en 9 se observa una MTUI; 4 presentan vejiga de alto riesgo con patrón de hiperactividad del detrusor terminal, baja capacidad y acomodación vesical y aumento de la actividad EMG durante la micción con residuo significativo (todos con RVU bilateral), 2 presentan hiperactividad del detrusor fásica, con acomodación vesical aceptable y aumento de la actividad EMG durante la micción con residuo (portadores de RVU unilateral) y 3 presentan vejiga de gran capacidad con hipoactividad del detrusor y aumento de la actividad EMG durante la micción con residuo significativo (uno con RVU bilateral).

\section{Relación con variables estudiadas}

La sintomatología urinaria no difiere en los distintos patrones de alteración urodinámica. Solo la incidencia de ITU muestra diferencias significativas entre los distintos patrones urodinámicos, siendo mayor en las niñas con patrón urodinámico tipo 4 (micción disfuncional)

\section{RELACION ENTRE MTUI Y LAS VARIABLES ESTUDIADAS}

Existencia de MTUI en el 86\% (54/63) de los pacientes de la población estudiada con RVU primario persistente. El porcentaje de alteración de la función del TUI al reclutamiento, por sexo, es del $96 \%$ en las niñas $(27 / 28)$ y del $77 \%$ en los varones (27/35); existiendo asociación estadísticamente significativa entre la existencia de MTUI y el sexo femenino con $\mathrm{p}<0,05$. (Tabla V).

La presencia de MTUl es significativamente mayor en los pacientes portadores de RVU bilateral, en ambos sexos; $100 \%$ en las niñas (10/10) y $92 \%$ en los varones (11/12). Existe asociación significativa entre la presencia de MTUI y la bilateralidad del RVU con $p<0,005$.

Considerando la población total estudiada, el porcentaje de MTUI es del $86 \%$ en el RVU grado II, 71\% en el RVU grado III, $90 \%$ en el RVU grado IV y $100 \%$ en el RVU grado V. Aunque existe una mayor tendencia a la afectación del TUI en los RVU de alto grado en ambos sexos, entre los varones es mas marcada la tendencia a presentar MTUI en los RVU unilaterales de alto grado respecto a los de grado intermedio $192 \%$ en los RVU de alto grado vs $37,5 \%$ en los RVU grado III). Estadísticamente, la presencia de MTUI es independiente del grado de RVU en ambos sexos, no existiendo asociación entre grado de RVU y MTUI.

Existe una asociación estadísticamente significativa entre la normalidad en la función del TUI y la ausencia de daño renal con $\mathrm{p}<0,05$. De otra forma, el $93 \%$ de los varones con MTUI (25/27) tienen daño renal asociado (Tabla III B). En la niña solo un $30 \%$ de las niñas con MTUI tienen daño renal asociado. Sin embargo, si consideramos la muestra de niñas con RVU bilateral de alto grado, la existencia de MTUI se correlaciona con la existencia de daño renal en el $80 \%$ de los casos (Tabla III A).

\section{DISCUSIÓN}

Este estudio representa una muestra de pacientes con RVU primario persistente, la mayoría de larga evolución, sin valoración urológica pediátrica previa (funcional y quirúrgica), cuyas características diferenciales son: la escasez de diagnósticos prenatales, la edad media elevada (global de 5,9 años; y de 7,7 años en las niñas), el porcentaje elevado de RVU de alto grado (59\%), de daño 
renal $(52 \%)$ y de malfunción del TUI (86\%), sin diferencias significativas entre varones y niñas. Este estudio representa una muestra poco frecuente en la actualidad, en la que el empleo sistematizado del tratamiento endoscopico del RVU ha reducido significativamente el número de pacientes con RVU de larga evolución, pero que puede reflejar y aportar datos en historia natural del RVU que lejos de remitir espontáneamente, ha persistido durante años.

En cuanto al sexo la muestra evidencia una homogeneidad en el número de pacientes del sexo masculino y femenino. Que el número de varones sea ligeramente superior al de niñas, probablemente se deba a la inclusión en el estudio de los casos nuevos de RVU -de diagnostico prenatal- durante el año del estudio prospectivo, la mayoría en lactantes varones.

Destaca la baja proporción de RVU de bajo grado en la población estudiada, quizá debido en parte al escaso número de diagnósticos prenatales, a la ausencia de intervención urológica en esta serie y a que son los RVU de menor gravedad los que con mayor probabilidad sufran resolución espontánea; probablemente la selección natural de los casos de RVU favorece la persistencia de los mas graves y complicados.
A pesar de la escasez de lactantes incluídos, en una muestra de edad media elevada y a pesar de excluír los casos con RVU bilateral fetal de alto grado con insuficiencia renal lo síndrome de válvulas de uretra posterior like), hallamos un $52 \%$ de pacientes de ambos sexos con daño renal asociado.

Desde que Rolleston en 1970 (8) describiera la afectación renal generalizada en los lactantes varones con reflujo masivo, muchos trabajos (Yeung, 1997) (9) han enfatizado la presencia de este tipo de daño renal (riñón pequeño displásico) de forma casi exclusiva en el varón con RVU fetal de alto grado. Sorprende en nuestra serie que el $50 \%$ de los riñones con afectación renal en las niñas (4 de 8 casos) sea de tipo generalizado con reducción significativa de la función renal diferencial gammagráfica (<40\%) incluyendo un caso de anulación funcional renal con riñón pequeño y displasia renal, sometido a nefrectomía retroperitoneoscopica, que fue diagnosticado desde la primoinfección urinaria. Si este caso es de etiología congénita, como el caso del lactante varón con anulación funcional renal unilateral de nuestra serie -que presentaba riñón pequeño displásico desde el nacimiento sin ITU previa, no lo podemos asegurar. Dentro de los patrones de afectación renal

\begin{tabular}{|c|c|c|c|c|c|c|c|c|}
\hline \multirow[t]{2}{*}{ GRADO } & \multirow[t]{2}{*}{ TOTAL } & \multirow[t]{2}{*}{ NIÑAS } & \multirow[t]{2}{*}{ VARONES } & \multicolumn{2}{|c|}{$\begin{array}{c}\text { NIÑAS CON RVU+DAÑO } \\
\text { RENAL }\end{array}$} & \multicolumn{2}{|c|}{$\begin{array}{l}\text { VARONES CON } \\
\text { RVU+DAÑO RENAL }\end{array}$} & \multirow[t]{2}{*}{$\begin{array}{l}\text { TOTAL RVU+ } \\
\text { DAÑO RENAL }\end{array}$} \\
\hline & & & & UNILAT & BILAT & UNILAT & BILAT & \\
\hline II & $\begin{array}{l}7 \\
(9 \text { UR) }\end{array}$ & $\begin{array}{l}5 \\
(7 \cup R)\end{array}$ & $\begin{array}{l}2 \\
(2 \text { UR) }\end{array}$ & & & & & \\
\hline III & $\begin{array}{l}17 \\
(21 U R)\end{array}$ & $\begin{array}{l}8 \text { (11UR) } \\
14\end{array}$ & $\begin{array}{l}9 \\
(10 \cup R)\end{array}$ & & & $3 / 8$ & & $3 / 8$ \\
\hline IV & $\begin{array}{l}29 \\
\text { (39 UR) }\end{array}$ & $\begin{array}{l}(18 \text { UR) } \\
1\end{array}$ & $\begin{array}{l}15 \\
(21 U R)\end{array}$ & $4 / 10$ & $3 / 4$ & $8 / 9$ & $5 / 6$ & $20 / 29$ \\
\hline V & $\begin{array}{l}10 \\
(16 \text { UR) }\end{array}$ & $\begin{array}{l}(2 \text { UR) } \\
28\end{array}$ & $\begin{array}{l}9 \\
\text { (14 UR) }\end{array}$ & & $1 / 1$ & $4 / 4$ & $5 / 5$ & $10 / 10$ \\
\hline TOTAL & $\begin{array}{l}63 \\
(85 \cup R)\end{array}$ & (38 UR) & $\begin{array}{l}35 \\
(47 \cup R)\end{array}$ & $\begin{array}{l}4 / 18 \\
(22 \%)\end{array}$ & $4 / 10$ & $\begin{array}{l}15 / 23 \\
(65 \%)\end{array}$ & $\begin{array}{l}10 / 12 \\
(83 \%)\end{array}$ & $33 / 63$ \\
\hline & & & & \multicolumn{2}{|c|}{$8 / 28(29 \%)$} & \multicolumn{2}{|c|}{ 25/35 (71\%) } & $(52 \%)$ \\
\hline
\end{tabular}


(desde el punto de vista anatomo-patológico) estan descritos, la asociación de cicatrices adicionales a la displasia renal -secundarias a ITU-, así como la displasia renal segmentaria que asemejan a la nefropatía por RVU adquirida típica del niño mayor (Ridson, 1993) (10). Si la preponderancia del sexo masculino es justificada por la selección de pacientes con RVU de alto grado al reclutamiento, como en la serie de Yeung (2006) (3), no sería de extrañar que los casos de niñas con lesiones similares aumentaran en las series con pacientes de dicho sexo y RVU de alto grado. Se baraja la causa anatómica de la configuración de la uretra en el varón, pero también interviene un factor congénito malformativo y genético.

En relación a ello, es destacable en el estudio el alto porcentaje de RVU de alto grado entre las niñas en nuestra población (54\%), que no difiere significativamente respecto a la cifra de los varones (69\%), a diferencia de lo descrito en series, en las que se afirma la excepcionalidad del RVU de alto grado y el daño renal generalizado en los pacientes del sexo femenino, en los que predominan los grados bajos y medios de RVU y la afectación renal focal. (Sillen, 1999) (11).
En este sentido, hemos observado, con asociación altamente significativa en nuestra serie, que el mayor grado de RVU está en relación directa con la presencia de daño renal, confirmando los hallazgos de otros autores (Bailey, 1973 (12), Swerkersson, 2007 (13).

Otra de las observaciones que pueden explicar estas diferencias radica en la asociación significativa entre la ausencia de malfunción del tracto urinario inferior y la ausencia de daño renal, acorde con otras series (Nielsen, 1989 (14), Homsy, 1994 (15), en las que existe una correlación significativa entre la incidencia y severidad de la nefropatía y la existencia de hiperactividad vesical, sobre todo en los RVU de alto grado, dando un significado pronóstico a la coexistencia de la MTUI con el RVU. En una población como la presentada en la que la proporción de MTUI y RVU es muy elevada $(86 \%)$ sobre todo en la población femenina (96\% de MTUI asociada), (con asociación significativa entre la presencia de MTUI y el sexo femenino), parece que la existencia de MTUI grave de larga evolución pudiera ser el factor diferencial y determinante de la coexistencia de RVU de alto grado y daño renal, incluso generalizado, tambien en el sexo femenino. Estudios recientes (Yeung, 2006 (3);

TABLA V. No DE PACIENTES CON RVU, SEGÚN GRADO, LADO AFECTACIÓN Y SEXO Y ASOCIACIÓN A MALFUNCIÓN DEL TRACTO URINARIO INFERIOR.

\begin{tabular}{|c|c|c|c|c|c|c|c|c|}
\hline \multirow[t]{2}{*}{ GRADO } & \multirow[t]{2}{*}{ TOTAL } & \multirow[t]{2}{*}{ NIÑAS } & \multirow[t]{2}{*}{ VARONES } & \multicolumn{2}{|c|}{$\begin{array}{c}\text { NIÑAS CON RVU+DAÑO } \\
\text { RENAL }\end{array}$} & \multicolumn{2}{|c|}{$\begin{array}{l}\text { VARONES CON } \\
\text { RVU+DAÑO RENAL }\end{array}$} & \multirow[t]{2}{*}{$\begin{array}{l}\text { TOTAL RVU+ } \\
\text { DAÑO RENAL }\end{array}$} \\
\hline & & & & UNILAT & BILAT & UNILAT & BILAT & \\
\hline II & (9 UR) & $\begin{array}{l}5 \\
(7 \cup R)\end{array}$ & $\begin{array}{l}2 \\
(2 \mathrm{UR})\end{array}$ & $3 / 3$ & $2 / 2$ & $1 / 2$ & - & $6 / 7$ \\
\hline III & $\mid \begin{array}{l}17 \\
(21 \text { UR) }\end{array}$ & $\begin{array}{l}8 \text { (11UR) } \\
14\end{array}$ & $\begin{array}{l}9 \\
(10 \cup R)\end{array}$ & $5 / 5$ & $3 / 3$ & $3 / 8$ & $1 / 1$ & $12 / 17$ \\
\hline IV & $\begin{array}{l}29 \\
(39 \text { UR) }\end{array}$ & $\begin{array}{l}(18 \text { UR) } \\
1\end{array}$ & $\begin{array}{l}15 \\
(21 U R)\end{array}$ & $9 / 10$ & $4 / 4$ & $8 / 9$ & $5 / 6$ & $26 / 29$ \\
\hline V & $\begin{array}{l}10 \\
(16 \text { UR) }\end{array}$ & $\begin{array}{l}(2 \text { UR) } \\
28\end{array}$ & $\begin{array}{l}9 \\
(14 \text { UR) }\end{array}$ & - & $1 / 1$ & $4 / 4$ & $5 / 5$ & $10 / 10$ \\
\hline \multirow[t]{2}{*}{ TOTAL } & $\begin{array}{l}63 \\
(85 \text { UR) }\end{array}$ & (38 UR) & $\begin{array}{l}35 \\
(47 \cup R)\end{array}$ & $17 / 18$ & $10 / 10$ & $16 / 23$ & $11 / 12$ & \multirow[t]{2}{*}{$54 / 63$} \\
\hline & & & & \multicolumn{2}{|c|}{$27 / 28$} & \multicolumn{2}{|c|}{$27 / 35$} & \\
\hline
\end{tabular}

Entre paréntesis, el no de unidades renales (UR). MTUI: Malfunción del TUI. UNILAT: Unilateral. BILAT: Bilateral. 
Godley, 2001 (16)| remarcan la correlación significativa entre la resolución espontanea del RVU y el estado de la función vesical y renal al diagnóstico, concluyendo que la coexistencia de MTUI y daño renal son factores predictivos de persitencia del RVU. Se propone una fuerte correlación entre el daño generalizado y la persistencia del RVU a largo plazo.

En la serie presentada queda patente que los RVU que persisten son los que se asocian a MTUl y daño renal graves, subrayando el importante papel que juega la función del TUI en la historia natural del RVU y su relación con el riesgo de daño renal. Koff en 1983 (17) subrayó lo contrario demostrando una mayor probabilidad de resolución del RVU en el grupo con hiperactividad (MTUI), sobre todo si esta era tratada medicamente, lo que también ensalza el papel de la función del TUI en la persistencia del RVU, sin tomar en consideración el daño renal.

Otros estudios subrayan que solo el $20 \%$ de los niños con RVU persistente presentan MTUI después de la edad de la adquisición normal de la continencia urinaria (Homayoon, 2005) (18). La muestra que presentan estos autores es, sin embargo, opuesta a la presente, con una gran mayoría de RVU de bajo grado (87\%) y función renal diferencial normal (82\%). El diagnóstico urodinámico lo basan en la presencia de síntomas (frecuencia e incontinencia) y signos exploratorios (ropa mojada), lo que también podría enmascarar la existencia de MTUI con respecto al diagnóstico urodinamico (no invasivo y cistométrico); estudio indicado para valorar la función del TUI.

A diferencia de los hallazgos de otros autores (Nielsen, 1989) (14) y, sobre todo, antes de la sistematización de la nomenclatura sobre la función del TUI (ICCS), hemos observado diferencias en los patrones de afectación urodinámica según el sexo, con exclusión de los lactantes varones incluídos en el estudio.

Destaca un predominio de la disfuncion miccional en las niñas con RVU, tal como hemos descrito previamente (19), que implica aumento de la contracción esfinteriana primaria durante la micción, hiperpresión del detrusor secundaria de mayor o menor gravedad, residuo postmiccional elevado, dificultad de vaciado o vaciado inefectivo e ITU, con asociación significativa entre la presencia de ITU y malfunción tipo micción disfuncional. En el varón, sin embargo, no hemos observado este patrón urodinámico, sino que predomina la hiperactividad del detrusor, de distinta gravedad (fásica o Terminal), con asociación a aumento de la contracción del esfínter durante la micción, secundario, aprendido y asociado a la existencia de CID, no constante, y que permite un vaciamiento efectivo vesical, en la mayoría de los casos. A excepción de la ITU no existen, sin embargo, diferencias significativas en relación a los síntomas de MTUI entre los distintos patrones de afectación urodinámica, ni en relación al sexo. En contra de los tópicos generales, que subrayan la asociación de urgenciaincontinencia diurna al patrón de hiperactividad vesical, frecuente en el varón, es común la asociación de urgencia e incontinencia diurna en las niñas con disfunción miccional, que postponen la micción como base de un hábito inadecuado miccional.
La valoración de la función de TUl es más difícil en el lactante en el que la MTUI no puede sospecharse en base a la presencia de síntomas tipo incontinencia (enuresis e incontinencia diurna) o urgencia. En este sentido, los parámetros de mayor relevancia a esta edad son: la presencia de ITU y el vaciamiento vesical incompleto (mediante la observación miccional durante 4 horas), con la observación del número e interrupción de micciones durante el período de observación. La elevada frecuencia de MTUl en el lactante varón con RVU de alto grado, bilateral, con daño renal, convierte a la afectación renal en un dato importante a valorar de sospecha de la presencia de MTUI; sugiriendo que no solo el daño renal sino la MTUI puedan ser de etiologia congénita $\circ$ estar geneticamente determinados en esta población. Los patrones urodinamicos que hemos observado en el estudio urodinámico de estos lactantes (la vejiga de alto riesgo con hiperactividad del detrusor y la vejiga de gran capacidad con elevado residuo postmiccional) podrían ser parte de una misma alteración, el segundo evolución del primero, con la caracteristica común de una dificultad de vaciado secundario a una micción no coordinada o descoordinación entre el esfínter y el detrusor.

En base a una clasificación práctica y descriptiva, se han dividido los patrones urodinámicos observados en dos grandes grupos; los que afectan a la fase de llenado y los que afectan a la fase miccional, sobre todo en base a la importante repercusión sobre el tratamiento a instaurar. Sin embargo, nos parece imposible valorar ambas fases por separado ya que el ciclo miccional es uno y los hallazgos en una fase tienen repercusión o son consecuencia de los hallazgos en la otra fase. El desengranaje de todos los hallazgos urodinamicos en ambas fases aumenta la precisión diagnóstica, así como resalta las claves de cada tratamiento individualizado.

La introducción y estandarización de las técnicas de antirreflujo endoscópico dentro del arsenal terapéutico del RVU han supuesto cambios, no solo en el abordaje y simplificación de la técnica quirúrgica -sin renunciar a la efectividad- (Capozza, 2007) (20), sino cambios en el manejo e indicación quirúrgica del RVU en la edad pediátrica.

Revisiones recientes de series de niños con RVU tratados endoscopicamente, en relación a su mayor o menor efectividad en presencia de malfunción del TUl (Lackgren, 2007) (21), siguen creando controversia acerca de si el RVU es causado o es la causa de la malfunción del TUI. Si el procedimiento endoscópico previene la ITU, mejorando la dinámica del TUI, está todavía sin demostrar. Pero no por ello se pueden ignorar los efectos de la malfunción del TUI sobre el tracto urinario superior (TUS), especialmente en los niños afectos de RVU e ITU. Así, la incontinencia urinaria no debe ser considerada como un síntoma simplemente molesto, independiente del fenómeno RVU, sino como un signo de potencial alteración de la función del TUI, que puede llevar consigo importantes implicaciones clínicas no solo actuales sino en el futuro. De igual forma, las formas severas del RVU con afectación grave generalizada de la función renal no deben ser consideradas solo como una displasia renal congénita asociada, sino como un "fenómeno RVU" en el que el RVU, la nefropatía y la MTUl están interrelacionadas, y cuyo origen se ha especu- 
lado recientemente pudiera ser común en forma de maldesarrollo embriológico de la yema ureteral y el trígono (Tanagho, 1965 (22), Yeung, 2006, (3).

\section{CONCLUSIONES}

Se subraya la relevancia de la realización del estudio urodinámico en el paciente con RVU en el hecho de que la función normal del TUI muestra una incidencia significativamente menor de daño renal o nefropatía por reflujo. Se sugiere que la MTUI subyacente pone en riesgo de ITU y nefropatía a los pacientes con RVU (quizá mal llamado "primario") de alto grado.

La realización de un despistaje no invasivo de la función del TUI mediante la historia miccional, el análisis urinario, la ecografía y la medida ecográfica del residuo postmiccional, permite realizar una selección de pacientes que se beneficiarán de un estudio urodinámico completo. Si existen síntomas o signos de MTUI en base a este screening, es recomendable la realización del estudio urodinámico completo. La cistometría pediátrica aporta datos precisos sobre las fases de llenado y vaciado vesical, que facilitan el tratamiento específico de la MTUI.

A diferencia de los niños con MTUI sin RVU asociado, el estudio urodinámico cobra un especial significado en el pronóstico de la malformación urológica asociada en los niños portadores del RVU.

Con esta sistemática de estudio de la función del TUl puede lograrse una detección precoz de los pacientes en riesgo, así como un tratamiento temprano, mediante una adecuada selección de los pacientes que se beneficiarán de la farmacoterapia y los que se beneficiarán de la uroterapia, con el fin de prevenir la progresión del daño renal y potenciar la curación definitiva del RVU.

\section{AGRADECIMIENTOS}

A la Dra. Ángeles Fernández, coordinadora de la Unidad de Nefrología Pediátrica del Servicio de Pediatría del Complejo Hospitalario de Toledo; gracias a su presentación de los pacientes, ha sido posible la realización de este estudio.

\section{BIBLIOGRAFÍA y LECTURAS RECOMENDADAS (*lectura de interés $y^{* *}$ lectura fundamental)}

*1. CAPOZZA, N.; LAIS, A.; MATARAZZO, E. y cols.: "Influence of voiding dysfunction on the outcome of endoscopic treatment for vesicoureteral reflux". J. Urol., 168: 1695, 2002.

2. HERNDON, C.D.; DECAMBRE, M.; McKENNA, P.H.: "Changing concepts concerning the management of vesicoureteral reflux". J. Urol., 166: 1439, 2001.

**3. YEUNG, C.K.; SREEDHAR, B.; SIHOE, J.D. y cols. "Renal and bladder functional status at diagnosis as predictive factors for the outcome of primary vesicoureteral reflux in children”. J. Urol., 176: 1152, 2006.
4. SILLÉN, U.; HOLMDAHL, G.; HELLSTRÖM, A.L. y cols.: "Treatment of bladder dysfunction and high grade vesicoureteral reflux does not influence the spontaneous resolution rate". J. Urol., 177: 325, 2007.

5. KOFF, S.A.: "Estimating bladder capacity in children". Urology, 21: 248, 1983.

6. LEBOWITZ, R.L.; OLBING, H.; PARKKULAINEN, K.V. y cols.: "International system of radiographic grading of vesicoureteric reflux. International reflux study in children". Pediatr. Radiol., 15: 105, 1985.

*7. NEVÉUS, T.; VON GONTARD, A.; HOEBEKE, P. y cols.: "The standardization of terminology of lower urinary tract function in children and adolescents: Report from the Standardisation Committee of the International Children's Continence Society”. J. Urol., 176: 314, 2006.

8. ROLLESTON, G.L.; SHANNON, F.T.; UTLEY, W.L.: "Relationship of infantile vesicoureteric reflux to renal damage". BMJ, 1: 460,1970.

9. YEUNG, C.K.; GODLEY, M.L.; DHILLON, H.K. y cols.: "The characteristics of primary vesico-ureteric reflux in male and female infants with prenatal hydronephrosis". Br. J. Urol., 80: 319, 1997.

10. RISDON, R.A.: "The small scarred kidney in childhood". Pediatr. Nephrol., 7: 361, 1993.

*11. SILLÉN, U.: "Vesicoureteral reflux in infants". Pediatr. Nephrol., 13: 355, 1999.

12. BAILEY, R.R.: "The relationship of vesico-ureteric reflux to urinary tract infection and chronic pyelonephritis-reflux nephropathy". Clin. Nephrol., 1: 132, 1973.

13. SWERKERSSON, S.; JODAL, U.; SIXT, R. y cols.: "Relationship among vesicoureteral reflux, urinary tract infection and renal damage in children". J. Urol., 178: 647, 2007.

14. NIELSEN, J.B.: "Lower urinary tract function in vesicoureteral reflux". Scand. J. Urol. Nephrol. Suppl., 125: 15, 1989.

15. HOMSY, Y.L.: "Dysfunctional voiding syndromes and vesicoureteral reflux". Pediatr. Nephrol., 8: 116, 1994.

16. GODLEY, M.L.; DESAI, D.; YEUNG, C.K. y cols.: "The relationship between early renal status, and the resolution of vesico-ureteric reflux and bladder function at 16 months". BJU Int., 87: 457, 2001.

17. KOFF, S.A.; MURTAGH, D.S.: "The uninhibited bladder in children: Effect of treatment on recurrence of urinary infection and on vesicoureteral reflux resolution”. J. Urol., 130: 1138, 1983.

18. HOMAYOON, K.; CHEN, J.J.; CUMMINGS, J.M. y cols.: "Voiding dysfunction: Outcome in infants with congenital vesicoureteral reflux". Urology, 66: 1091, 2005.

*19. MARTÍN-CRESPO IZQUIERDO, R.; LUQUE MIALDEA, R.: "Non-coordinated micturition syndrome mimicking posterior urethral valves in a male neonate". Cir. Pediatr., 16: 134, 2003.

20. CAPOZZA, N.; CAIONE, P.: "Vesicoureteral reflux: Surgical and endoscopic treatment". Pediatr. Nephrol., 22: 1261, 2007.

21. LÄCKGREN, G.; SKÖLDENBERG, E.; STENBERG, A.: "Endoscopic treatment with stabilized nonanimal hyaluronic acid/dextranomer gel is effective in vesicoureteral reflux associated with bladder dysfunction". J. Urol., 177: 1124, 2007.

22. TANAGHO, E.A.; HUTCH, J.A.; MEYERS, F.H. y cols.: "Primary vesicoureteral reflux: Experimental studies of its etiology". J. Urol., 93: 165, 1965. 